\title{
ORIGINAL
}

\section{PROBABILIDAD DE ÉXITO EN EL ABANDONO DEL TABACO EN EL CURSO DE DOS INTERVENCIONES SENCILLAS PARA DEJAR DE FUMAR (*)}

Pilar Guallar-Castillón (1), Pedro Lafuente Urdinguio (2), Peru Garteizaurrekoa Dublang (3), Olga Sáinz Martínez (3), José I. Díez Azcárate (4), Manuel Foj Alemán (4), Jose R. Banegas Banegas (1), Victoria Prado Méndez (2), Belén de Andrés Manzano (1) y Fernando Rodríguez-Artalejo (1)

(1) Departamento de Medicina Preventiva y Salud Pública. Universidad Autónoma de Madrid. Madrid.

(2) Departamento de Medicina Preventiva y Salud Pública. Universidad del País Vasco. Bilbao.

(3) Unidad de Salud Laboral. Empresa de Transportes Colectivos de Bilbao, S.A. Bilbao.

(4) Unidad de Salud Laboral. Iberdrola, S.A. Bilbao.

(*) Este trabajo se ha financiado parcialmente con la ayuda de la Universidad del País Vasco 004. EA079/98. Los parches de nicotina se han proporcionado gratuitamente por Novartis España. Los autores son responsables únicos e independientes del contenido del artículo, y de la decisión de su publicación

\section{RESUMEN}

Fundamentos: El consejo antitabaco dirigido a los fumadores mejoraría si se les informa de las posibilidades de éxito en el abandono del tabaco a lo largo del tiempo desde el inicio de la cesación tabáquica. Este trabajo mide el cambio en la probabilidad de éxito tabáquica. Este trabajo mide el cambio en la probabilidad de éxito
en el abandono del tabaco durante el seguimiento de dos intervenciones sencillas para dejar de fumar.

Métodos: Estudio prospectivo en tres centros laborales de Vizcaya. La intervención 1 consistió en consejo médico esporádico, no estructurado y de breve duración (30 segundos-1 minuto). La intervención 2 consistí en consejo estucturado en la prime La visita (5-8 minutos), consejo de refuerzo en tres ocasiones durante 3 meses, y parches de nicotina administrados individualizadamente según el grado de dependencia nicotínica. La medida de éxito de la intervención fue una razón de proporciones (RP), formada por el valor predictivo positivo de abstinencia tabáquica a los 12 meses entre los que no fumaban a los 2 días, a los 15 días y a los 3 meses del inicio de la intervención, dividido por la proporción de sujetos que no fumaban a los 12 meses.

Resultados: Para los 103 fumadores con la intervención 1, la RP fue 1,7 (IC 95\%: 1,0-3,4) a los 2 días; $2,3(1,2-4,6)$ a los 15 días, y $3,4(1,8-6,5)$ a los 3 meses. Para los 114 fumadores con la intervención 2 , la RP fue $1,3(1,0-2,1)$ a los 2 días; $1,9(1,2-2,8)$ a los 15 días, y $2,6(1,8-3,8)$ a los 3 meses. Los resultados no cambiaron sustancialmente al estratificar por el número de cigarrillos o el grado de dependencia nicotínica.

Conclusiones: No fumar a los 3 meses del inicio de una intervención para dejar de fumar aumenta sustancialmente la probabilidad de éxito a los 12 meses.

Palabras clave: Cese del uso de tabaco. Programación. Tabaco. Valor predictivo.

Correspondencia:

Fernando Rodríguez-Artalejo

Departamento de Medicina Preventiva y Salud Pública.

Facultad de Medicina.

Universidad Autónoma de Madrid.

Avda. Arzobispo Morcillo, s/n

28029 Madrid

Correo electrónico: fernando.artalejo@uam.es

\section{ABSTRACT}

Probability of success in tobacco quitting during the course of two simple medical interventions

Introduction and objectives: Counselling for tobacco quitting could be improved by informing smokers about the likelihood of success in quitting over time since the start of an intervention for smoking cessation. This study assessed the change in the probability of success in tobacco quitting during the course of two simple medical interventions for smoking cessation.

Methods: Prospective study in three worksites in the Basque Country (Spain), which underwent two interventions for quitting. Intervention 1 consisted of sporadic, brief ( 30 seconds- 1 minute) and unstructured medical advice to quit. Intervention 2 consisted of brief (5-8 minutes) structured medical councelling for tobacco quitting in a firts visit, followed by reinforcement advice on 3 occasion during 3 months, accompanied by nicotine patches individualized according to nicotine dependence. Results are expressed as a «relative measure of success» or rate of proportions (RP) calculated as the predictive value of achieving tobacco abstinence at 12 months for those with tobacco abstinence at 2 days, 15 days and 3 months from the start of each intervention divided by the percentage abstinent at 12 .

Results: For the 103 smokers subjected to intervention 1, RP was 1.7 (CI 95\%: 1.0-3.4) at 2 days, $2.3(1.2-4.6)$ at 15 days, and 3.4 (1.8-6.5) at 3 months. For the 114 subjects who underwent intervention 2, RP was $1.3(1.0-2.1)$ at 2 days, $1.9(1.2-2.8)$ at 15 days, and $2.6(1.8-3.8)$ at 3 months. Results did not change materially after stratification by number of cigarettes smoked, or nicotine dependence.

Conclusions: Remaining abstinent from tobacco smoking at 3 monts after the start of a quitting intervention increases the likelihood of success in quitting at 12 months.

Key words: Tobacco. Tobacco use cessation. Predictive value. Programming. 


\section{INTRODUCCIÓN}

El consejo médico estructurado y de breve duración es eficaz para dejar de fumar ${ }^{1,2}$, y también lo es su combinación con tratamiento sustitutivo de la nicotina ${ }^{3}$. Además, parece haber una relación dosis-respuesta entre la intensidad del consejo médico y su efectividad ${ }^{4}$, de forma que proporcionar niveles intensos de apoyo a los fumadores puede aumentar la probabilidad de dejar el tabaco $^{2}$.

Aunque en los últimos años se han desarrollado guías de práctica clínica y programas que facilitan la realización del consejo para dejar de fumar ${ }^{4-6}$, los médicos no siempre proporcionan un consejo completo y ofrecen visitas de seguimiento ${ }^{7-10}$. En estas guías se recomienda a los médicos que ofrezcan a los fumadores visitas de seguimiento en las primeras semanas de cesación tabáquica, ya que ellas aumentan las expectativas de éxito porque ayuda a superar un período crítico en el que el deseo de fumar es más intenso ${ }^{11}$. Además, no vencer el deseo de fumar es el motivo más frecuente de recaída en el consumo de tabaco ${ }^{12}$. Por tanto, puede ser útil que el médico conozca y mejore el contenido del consejo antitabaco a los fumadores en la fase de seguimiento.

El objetivo de este trabajo es medir el cambio en la probabilidad de dejar de fumar a los 12 meses de una intervención, según pasa el tiempo que el sujeto ha permanecido sin fumar desde el inicio de la misma. Ello se calcula para dos intervenciones sencillas de cesación tabáquica, con el fin de que esta información pueda incorporarse al consejo médico para dejar de fumar.

\section{SUJETOS Y MÉTODOS}

Los datos se han obtenido de un ensayo clínico randomizado que evaluó dos intervenciones para dejar de fumar en los trabajadores de tres centros laborales de Vizcaya: la empresa de Transportes Colectivos de Bilbao, cuyos trabajadores son mayoritariamente conductores de autobuses, y los centros de Gardoqui y Larrasquitu de la empresa eléctrica Iberdrola, cuyos trabajadores se dedican principalmente a actividades administrativas. Los datos del estudio fueron recogidos por el médico de empresa de los tres centros laborales donde se realizó el estudio, y en uno de ellos además por una enfermera. El ensayo clínico pretendía evaluar la eficacia de una estrategia individualizada para dejar de fumar adaptada al grado de dependencia nicotínica de cada fumador. El estudio fue aprobado por el Comité Ético de Investigación Clínica del Hospital de Basurto de Bilbao. Información adicional sobre este ensayo puede encontrarse en la literatu$\mathrm{ra}^{13}$.

Se estudió a 217 sujetos de 18 a 63 años de edad que habían fumado cigarrillos durante el último mes, tenían una concentración de monóxido de carbono $(\mathrm{CO})$ en aire espirado > 10 ppm en el momento del examen médico, y estaban motivados para dejar de fumar de acuerdo al test de Richmond ${ }^{14}$.

La intervención 1 era la práctica habitual en los centros laborales y consistió en realizar esporádicamente un consejo médico verbal no estructurado de breve duración (de 30 segundos a un minuto). La intervención 2 incluía una breve sesión (5-8 minutos) de consejo estructurado e individualizado basado en el material elaborado por el Instituto Nacional del Cáncer de Estados Unidos $^{11}$. El consejo terminaba con la entrega de un folleto sobre cesación tabáquica ${ }^{15}$. Algunos participantes recibieron además tratamiento con parches de nicotina según su dependencia nicotínica medida con el test de Fagerström (TF) ${ }^{16}$. Una mayor puntuación en el TF indica mayores niveles de dependencia. Si el TF fue menor o igual a 4, la intervención consistió sólo en consejo médico individualizado; para un TF de 5 a 7 la intervención incluyó consejo médico individualizado y parches de nicotina (Nicoti- 
nell TTS, Novartis Consumer Health) de 14 $\mathrm{mg}$ /día durante 8 semanas, seguidos de parches de $7 \mathrm{mg}$ /día durante 4 semanas; y cuando el TF tuvo una puntuación mayor de 7 la intervención consistió en consejo médico individualizado y parches de nicotina de $21 \mathrm{mg} /$ día durante 4 semanas, seguidos de parches de $14 \mathrm{mg} /$ día durante 4 semanas, y terminó con parches de $7 \mathrm{mg}$ /día durante 4 semanas. En total 62 sujetos recibieron parches de nicotina. La intervención 2 se prolongó con tres nuevos contactos en la fase de seguimiento: a los 2 días de la fecha prevista para dejar de fumar, a los 15 días y a los 3 meses. En estas visitas se realizó un breve consejo (2-3 minutos), con el que se reforzó la decisión de abandono del tabaco, y se entrenó al participante en el manejo de situaciones difíciles relacionadas con la abstinencia tabáquica.

La abstinencia tabáquica se evaluó mediante entrevista y se confirmó con la determinación de $\mathrm{CO}$ en aire espirado (10 ppm) en el momento de la visita (micro-Smokerlyzer de Bedfont Technical Instruments). En concreto, la cesación tabáquica se evaluó recogiendo datos de abstinencia puntual a los 2 días, a los 15 días, a los 3 meses y a los 12 meses de la fecha prevista para dejar de fumar.

Se adoptaron un conjunto de medidas para reducir la variabilidad interobservador y entre los tres centros del estudio. Primero, cada una de las intervenciones estaba estandarizada en el manual operacional del estudio. Segundo, todas las cuestiones a incluir en el cuaderno de recogida de datos tenían formato cerrado. Tercero, todos los médicos y la enfermera de los centros del estudio participaron en un programa de entrenamiento sobre la intervención y la recogida de datos. Por último, el estudio fue sometido a monitorización clínica para asegurar que dichos procesos y, en particular, la recogida de datos fuera completa y correcta. Además, no existieron pérdidas en el seguimiento de los sujetos, dada la alta motiva- ción de los investigadores de las unidades de salud laboral de los 3 centros para el seguimiento de los trabajadores del estudio, y que éstos son una población con alta estabilidad en el empleo.

Para las dos intervenciones de abandono tabáquico, se calcularon el valor predictivo positivo (VPP) o probabilidad de abstinencia tabáquica a los 12 meses si se ha dejado de fumar en cada visita de seguimiento, y el valor predictivo negativo (VPN) o probabilidad de fumar a los 12 meses si el participante fumaba en cada visita de seguimiento. El VPP y el VPN se acompañaron de sus respectivos intervalos de confianza al 95\% ${ }^{17}$. También se calculó una medida relativa de éxito, calculada como la razón del VPP en cada visita sobre el porcentaje de sujetos que se espera hayan dejado el tabaco a los 12 meses de la intervención, estimado al principio de la misma (porcentaje de personas que no fuman a los 12 meses entre todos los fumadores sometidos a la intervención). Esta medida relativa de éxito es por construcción una razón de proporciones (RP), y se ha calculado junto a sus intervalos de confianza al $95 \%{ }^{17}$.

Se debe destacar que el análisis descrito más arriba se ha realizado a partir de los datos de un ensayo clínico, pero no tiene naturaleza comparativa entre las dos ramas o intervenciones del mismo. En otras palabras, el análisis trata los datos como si procediesen de un estudio observacional en el que los sujetos sometidos a las dos intervenciones son considerados como dos cohortes independientes que no se comparan directamente entre sí.

\section{RESULTADOS}

Participaron en el estudio 217 fumadores, de los cuales 103 recibieron consejo médico esporádico no estructurado (intervención 1) y 114 recibieron consejo estructurado y parches de nicotina según el grado de depen- 
Tabla 1

Características de los sujetos que recibieron las dos intervenciones para dejar de fumar

\begin{tabular}{|c|c|c|}
\hline & $\begin{array}{c}\text { Intervención } 1^{*} \\
n=103\end{array}$ & $\begin{array}{c}\text { Intervención } 2^{* *} \\
n=114\end{array}$ \\
\hline \multicolumn{3}{|l|}{ Datos sociodemográficos } \\
\hline Edad (años) & $43,3 \pm 8,3$ & $43,1 \pm 8,2$ \\
\hline Sexo (\% varones) & 85,4 & 87,0 \\
\hline \multicolumn{3}{|l|}{ Nivel de estudios: } \\
\hline Sin estudios $(\%)$ & 24,3 & 23,5 \\
\hline Estudios primarios $(\%)$ & 17,5 & 16,5 \\
\hline Estudios secundarios $(\%)$ & 33,0 & 39,1 \\
\hline Estudios universitarios (\%) & 25,2 & 20,9 \\
\hline Índice de masa corporal $\left(\mathrm{kg} / \mathrm{m}^{2}\right)$ & $27,2 \pm 4,5$ & $26,4 \pm 3,8$ \\
\hline \multicolumn{3}{|l|}{ Hábitos y consumo tabáquico } \\
\hline Cigarrillos día & $27,6 \pm 11,7$ & $25,0 \pm 10,6$ \\
\hline CO espirado (ppm) & $37,0 \pm 19,0$ & $37,0 \pm 17,5$ \\
\hline \multicolumn{3}{|l|}{ Motivos para dejar de fumar } \\
\hline Si fuera fácil dejaría de fumar con alta probabilidad (\%) & 97,1 & 100 \\
\hline Con deseo fuerte o muy fuerte de dejar de fumar (\%) & 78,6 & 76,5 \\
\hline Es seguro o muy probable que en este intento dejará de fumar (\%) & 57,3 & 52,2 \\
\hline Dejar de fumar será fácil o muy fácil (\%) & 8,7 & 13,0 \\
\hline Existen motivos de salud para dejar de fumar o se lo prescribe el médico (\%) & 86,4 & 92,2 \\
\hline \multicolumn{3}{|l|}{ Historia de consumo e intentos de cesación } \\
\hline Edad de inicio (años) & $16,6 \pm 4,0$ & $16,5 \pm 3,3$ \\
\hline Duración del hábito (años) & $26,6 \pm 8,6$ & $26,9 \pm 8,6$ \\
\hline En los últimos 5 años ha pensado seriamente dejar de fumar (\%) & 78,6 & 84,3 \\
\hline \multicolumn{3}{|l|}{ En los últimos 5 años ha intentado dejar de fumar: } \\
\hline Nunca (\%) & 35,3 & 41,7 \\
\hline Una vez $(\%)$ & 21,6 & 32,2 \\
\hline Dos o tres veces $(\%)$ & 28,4 & 19,1 \\
\hline Cuatro o más veces $(\%)$ & 14,7 & 7,0 \\
\hline \multicolumn{3}{|l|}{ Tiempo máximo que permanece sin fumar entre los que lo intentan } \\
\hline Menos de 2 semanas $(\%)$ & 49,2 & 42,6 \\
\hline Entre 2 semanas y 6 meses $(\%)$ & 35,4 & 35,3 \\
\hline Más de 6 meses (\%) & 15,4 & 22,1 \\
\hline \multicolumn{3}{|l|}{ Entorno social } \\
\hline Pasa la mayor parte de su tiempo con otros fumadores (\%) & 52,4 & 53,9 \\
\hline Tiene pareja fumadora $(\%)$ & 37,9 & 42,6 \\
\hline Tiene familiares de primer grado fumadores $(\%)$ & 88,3 & 86,1 \\
\hline En su jornada laboral pasa más tiempo con fumadores (\%) & 21,4 & 19,1 \\
\hline En el tiempo de ocio pasa más tiempo con fumadores (\%) & 43,7 & 47,0 \\
\hline \multicolumn{3}{|l|}{ Dependencia } \\
\hline Puntuación de la prueba de Fagerström & $5,1 \pm 2,8$ & $4,5 \pm 2,4$ \\
\hline Grado I (Fagerström $\leq 4)(\%)$ & 39,8 & 42,6 \\
\hline Grado II (Fagerström 5-7) (\%) & 36,9 & 45,2 \\
\hline Grado III (Fagerström $\geq 8)(\%)$ & 23,3 & 12,2 \\
\hline
\end{tabular}

Los valores \pm representan media \pm desviación estándar.

* Intervención 1: Consejo médico esporádico no estructurado, esporádico y de breve duración (30 segundos a un minuto). **Intervención 2: Consejo estructurado e individualizado (5-8 minutos), entrega de un folleto sobre cesación tabáquica, consejo de refuerzo en tres ocasiones durante tres meses y tratamiento con parches de nicotina según su dependencia nicotínica.

dencia (intervención 2). Fueron en su mayoría varones con una edad media de 43 años, que fumaban como media más de 20 cigarrillos al día y llevaban más de 25 años fumando. Las características sociodemográficas y en relación con el hábito tabáquico aparecen en la tabla 1.
En los fumadores sometidos a la intervención 1, el porcentaje de sujetos que consiguió la abstinencia a los 12 meses fue del 13,6\%. El VPP de abstinencia aumentó en cada visita, llegando a ser del $45 \%$ a los 3 meses. La RP de abstinencia tabáquica fue 1,7 (IC 95\%: 1,0-3,4) a los 2 días de la in- 
Tabla 2

Porcentaje de sujetos que consiguen la abstinencia, valor predictivo positivo (VPP), valor predictivo negativo (VPN) y razón de proporciones (RP) de abstinencia tabáquica para dos intervenciones para dejar de fumar

\begin{tabular}{|c|c|c|c|c|c|c|c|c|}
\hline & \multicolumn{2}{|c|}{ Porcentaje de abstinencia } & \multicolumn{2}{|c|}{ Valor Predictivo Positivo (\%) } & \multicolumn{4}{|c|}{ Valor Predictivo Negativo(\%) Razón de Proporciones } \\
\hline & $\%$ & IC $95 \%$ & $V P P$ & IC $95 \%$ & $V P N$ & IC $95 \%$ & $R P$ & IC $95 \%$ \\
\hline \multicolumn{9}{|l|}{ Intervención 1} \\
\hline Visita 2 días & 53,4 & $43,3-63,3$ & 23,6 & $13,3-37,0$ & 97,9 & $88,9-99,9$ & 1,7 & $1,0-3,4$ \\
\hline Visita 15 días & 34,0 & $24,9-44,0$ & 31,4 & $16,9-49,3$ & 95,6 & $87,6-99,1$ & 2,3 & $1,2-4,6$ \\
\hline Visita 3 meses & 23,3 & $15,5-32,7$ & 45,8 & $25,6-67,2$ & 96,2 & $89,3-99,2$ & 3,4 & $1,8-6,5$ \\
\hline Visita 12 meses & 13,6 & $7,6-21,8$ & & & & & & \\
\hline \multicolumn{9}{|l|}{ Intervención 2} \\
\hline Visita 2 días & 64,9 & $55,4-73,6$ & 35,1 & $24,4-47,1$ & 90,0 & $76,3-97,2$ & 1,3 & $1,0-2,1$ \\
\hline Visita 15 días & 46,5 & $37,1-56,1$ & 49,1 & $35,1-63,2$ & 93,4 & $84,1-98,2$ & 1,9 & $1,2-2,8$ \\
\hline Visita 3 meses & 34,2 & $25,6-43,7$ & 69,2 & $52,4-83,0$ & 96,0 & $88,8-99,2$ & 2,6 & $1,8-3,8$ \\
\hline Visita 12 meses & 26,3 & $18,5-35,4$ & & & & & & \\
\hline
\end{tabular}

Intervención 1: ver tabla 1; Intervención 2: ver tabla 1; VPP: probabilidad de no fumar a los 12 meses si no se fuma en esa visita; VPN: probabilidad de fumar a los 12 meses si se fuma en esa visita; RP: VPP en esa visita dividido por el porcentaje de sujetos que no fuman a los 12 meses de la intervención.

tervención, 2,3 (IC 95\%: 1,2-4,6) a los 15 días, y 3,4 (IC 95\%: 1,8-6,5) a los 3 meses. Además el VPN en cada visita fue superior al $95 \%$ (tabla 2).

Para los sujetos sometidos a la intervención 2, el porcentaje de sujetos que dejaron de fumar a los 12 meses fue de $26,3 \%$. El VPP fue superior al de la estrategia anterior y alcanzó el $69 \%$ a los 3 meses. La RP fue 1,3 (IC 95\%: 1,0-2,1) a los 2 días de la abstinencia, 1,9 (IC 95\%: 1,2-2,8) a los 15 días, y 2,6 (IC 95\%: 1,8-3,8) a los 3 meses. El VPN en cada visita fue superior al $90 \%$ (tabla 2).

Los resultados no variaron sustancialmente al desagregarlos según el número de cigarrillos consumidos y el grado de dependencia nicotínica medido por el TF, siendo los VPP mayores en la intervención 2 que en la intervención 1. Es de destacar que, en la intervención 2, el VPP a los 3 meses en sujetos que fumaban más de 20 cigarrillos/día fue del $72 \%$, y que entre los que tenían mayor dependencia fue del 75\% (tabla 3 ).

\section{DISCUSIÓN}

Nuestros resultados muestran que la probabilidad de no fumar a los 12 meses de la fecha prevista para dejar de fumar aumenta a lo largo del tiempo entre los que han dejado de fumar en cada visita de seguimiento. En comparación con la probabilidad de dejar de fumar esperada al inicio de la intervención, la abstinencia tabáquica a los 15 días de la fecha prevista para dejar de fumar aumenta aproximadamente dos veces la probabilidad de no fumar a los 12 meses, y la abstinencia a los 3 meses la aumenta tres veces. A diferencia de los VPP que fueron superiores en la intervención 2 como consecuencia del consejo estructurado y los parches de nicotina, las RP fueron similares en las dos intervenciones y para diferentes consumos de cigarrillos y grado de dependencia nicotínica. Ya que, a diferencia de los valores predictivos, el valor de la RP no depende de la tasa de cesación tabáquica (porque ajusta por ella al colocarla en el denominador de la razón), los resultados de la RP se pueden generalizar más fácilmente a otros contextos (centros de trabajo, centros de atención primaria) o a intervenciones de distinta eficacia absoluta. De ahí el interés de presentar en este trabajo los resultados en forma de RP.

En otros estudios en países anglosajones también se ha observado que no fumar en la primera semana ${ }^{18,19}$ es un buen predictor 
Tabla 3

Porcentaje de sujetos que consiguen la abstinencia, valor predictivo positivo (VPP) y razón de proporciones (RP) de abstinencia tabáquica para dos intervenciones para dejar de fumar, según consumo de cigarrillos y grado de dependencia nicotínica

\begin{tabular}{|c|c|c|c|c|c|c|}
\hline & \multicolumn{2}{|c|}{ Porcentaje de abstinencia } & \multicolumn{2}{|c|}{ Valor Predictivo Positivo (\%) } & \multicolumn{2}{|c|}{ Razón de Proporciones } \\
\hline & $\%$ & IC $95 \%$ & $V P P$ & IC $95 \%$ & $R P$ & IC $95 \%$ \\
\hline \multicolumn{7}{|l|}{ Intevención 1} \\
\hline \multicolumn{7}{|c|}{$N .^{0}$ de cigarrillos $\leq 20 /$ día $^{2}$} \\
\hline Visita 2 días & 52,5 & $36,1-68,5$ & 23,8 & $8,2-47,2$ & 1,6 & $1,0-4,6$ \\
\hline Visita 15 días & 35,0 & $20,6-51,7$ & 35,7 & $12,8-64,9$ & 2,4 & $1,0-6,6$ \\
\hline Visita 3 meses & 25,0 & $12,7-41,2$ & 40,0 & $12,2-70,9$ & 2,7 & $1,0-7,7$ \\
\hline Visita 12 meses & 15,0 & $5,7-29,8$ & & & & \\
\hline \multicolumn{7}{|c|}{$N{ }^{\circ}$ de cigarrillos $>20 /$ día } \\
\hline Visita 2 días & 54,0 & $40,9-66,6$ & 23,5 & $10,7-41,2$ & 1,9 & $0,8-4,5$ \\
\hline Visita 15 días & 33,3 & $22,0-46,3$ & 28,6 & $11,3-52,2$ & 2,3 & $0,9-5,7$ \\
\hline Visita 3 meses & 22,2 & $12,7-34,5$ & 50,0 & $23,0-77,0$ & 3,9 & $1,7-9,1$ \\
\hline Visita 12 meses & 12,7 & $5,7-23,5$ & & & & \\
\hline \multicolumn{7}{|l|}{ Test de Fagerström $<5$} \\
\hline Visita 2 días & 61,0 & $44,5-75,8$ & 28,0 & $12,1-49,4$ & 1,4 & $1,0-3,5$ \\
\hline Visita 15 días & 36,6 & $22,1-53,1$ & 40,0 & $16,3-67,7$ & 2,1 & $1,0-4,9$ \\
\hline Visita 3 meses & 26,8 & $14,2-41,8$ & 45,5 & $16,7-76,6$ & 2,3 & $1,0-5,7$ \\
\hline Visita 12 meses & 19,51 & $8,8-34,9$ & & & & \\
\hline \multicolumn{7}{|c|}{ Test de Fagerström $\geq 5$} \\
\hline Visita 2 días & 48,4 & $35,5-61,4$ & 20,0 & $7,7-38,6$ & 2,1 & $1,0-5,9$ \\
\hline Visita 15 días & 32,3 & $20,9-45,3$ & 25,0 & $8,7-49,1$ & 2,6 & $1,0-7,6$ \\
\hline Visita 3 meses & 21,0 & $11,7-33,2$ & 46,2 & $19,2-74,9$ & 4,8 & $1,8-12,5$ \\
\hline Visita 12 meses & 9,68 & $3,6-19,9$ & & & & \\
\hline \multicolumn{7}{|l|}{ Intervención 2} \\
\hline \multicolumn{7}{|c|}{$N .^{\circ}$ de cigarrillos $\leq 20 /$ día } \\
\hline Visita 2 días & 61,4 & $47,6-74,0$ & 28,6 & $14,6-46,3$ & 1,5 & $1,0-3,1$ \\
\hline Visita 15 días & 38,6 & $26,0-52,4$ & 36,4 & $17,2-59,3$ & 1,9 & $1,0-4,1$ \\
\hline Visita 3 meses & 24,6 & $14,1-37,8$ & 64,3 & $35,1-85,7$ & 3,3 & $1,7-6,4$ \\
\hline Visita 12 meses & 19,3 & $10,0-31,9$ & & & & \\
\hline \multicolumn{7}{|c|}{$N{ }^{\circ}$ de cigarrillos $>20 /$ día } \\
\hline Visita 2 días & 68,4 & $54,8-80,1$ & 41,0 & $25,6-57,9$ & 1,2 & $1,0-2,1$ \\
\hline Visita 15 días & 54,4 & $40,7-67,6$ & 58,1 & $39,1-75,5$ & 1,7 & $1,1-2,8$ \\
\hline Visita 3 meses & 43,9 & $30,7-57,6$ & 72,0 & $50,6-87,9$ & 2,2 & $1,4-3,4$ \\
\hline Visita 12 meses & 33,3 & $21,4-47,1$ & & & & \\
\hline \multicolumn{7}{|l|}{ Test de Fagerström $<5$} \\
\hline Visita 2 días & 65,3 & $50,4-78,3$ & 37,5 & $21,1-56,3$ & 1,3 & $1,0-2,5$ \\
\hline Visita 15 días & 40,8 & $27,0-55,8$ & 55,0 & $31,5-76,9$ & 1,9 & $1,1-3,5$ \\
\hline Visita 3 meses & 38,8 & $25,2-53,8$ & 63,2 & $38,4-83,7$ & 2,2 & $1,3-3,9$ \\
\hline Visita 12 meses & 28,6 & $16,6-43,3$ & & & & \\
\hline \multicolumn{7}{|l|}{ Test de Fagerström $\geq 5$} \\
\hline Visita 2 días & 64,6 & $51,8-76,1$ & 33,3 & $19,6-49,5$ & 1,4 & $1,0-2,5$ \\
\hline Visita 15 días & 50,8 & $38,1-63,4$ & 45,5 & $28,1-63,6$ & 1,9 & $1,1-3,3$ \\
\hline Visita 3 meses & 30,8 & $19,9-43,4$ & 75,0 & $50,9-91,3$ & 3,1 & $1,9-5,0$ \\
\hline Visita 12 meses & 24,6 & $14,8-36,9$ & & & & \\
\hline
\end{tabular}

Intervención 1: ver tabla 1; Intervención 2: ver tabla 1; VPP: probabilidad de no fumar a los 12 meses si no se fuma en esa visita; RP: VPP en esa visita dividido por el porcentaje de sujetos que no fuman a los 12 meses de la intervención.

del éxito al año del inicio de la intervención. En España, sólo se ha publicado hasta ahora el valor predictivo de la cesación tabáquica a los 2 meses del seguimiento ${ }^{20}$, obteniéndose valores comparables a los nuestros. Conocer la probabilidad de éxito al principio del seguimiento (por ejemplo a los 15 días) del sujeto que intenta dejar de fumar es especialmente importante en España por varias razones que ilustran el ambiente ad- verso para dejar de fumar en nuestro país y, por tanto, la dificultad para conseguirlo. Primero, por tener una de las prevalencias de consumo de tabaco más altas de Europa, estimándose que ésta es del $35,8 \%$, y la de grandes fumadores del $15,5 \%{ }^{21}$. Segundo, por la gran tolerancia social con el consumo de tabaco; más de la mitad de las personas que iniciaron el estudio pasaban la mayor parte del tiempo con otros fumadores, y más 
del $85 \%$ tenían familiares de primer grado fumadores (tabla 1). Tercero, porque España presenta muy poco cumplimiento de las políticas poblacionales contra el tabaco ${ }^{22}$.

Para los fumadores que inician la cesación tabáquica puede resultar alentador el informarles de que incluso a los 15 días de abstinencia la probabilidad de éxito es mucho mayor que el primer día, y que esta probabilidad aumentará aún más si en la próxima visita de seguimiento continúan sin fumar. También resulta estimulante que los fumadores sometidos a la intervención 2 tienen una alta probabilidad de no fumar a los 12 meses si lo han dejado a los 3 meses (tabla 3), como consecuencia de la eficacia del tratamiento, incluso en el caso de aquellos fumadores con más dificultad para dejar el hábito por un mayor nivel de dependencia nicotínica o por la mayor cantidad de cigarrillos consumidos. Esto tiene especial interés porque un mayor nivel de dependencia nicotínica es un predictor negativo de cesación tabáquica ${ }^{20,23,24}$.

Al igual que en otros estudios ${ }^{19,20}$, el VPN para las dos intervenciones fue muy alto en todas las visitas. Por tanto, los sujetos que fuman en el momento de una visita tienen muy pocas probabilidades de conseguir dejar de fumar a los 12 meses. El VPN es muy elevado no sólo a los 2 meses como encuentran otros autores ${ }^{20}$, sino desde los primeros días del seguimiento. Esto es importante porque desde ese momento ya se puede reorientar el consejo médico e incluso interrumpir el tratamiento sustitutivo de la nicotina, pues muy probablemente no se derivarán beneficios del mismo. Sin embargo, se debe ofrecer a los fumadores la posibilidad de apoyo si en un futuro desean intentar dejar de fumar otra vez. De hecho, el $66 \%$ de los que dejaron de fumar en la intervención 1 , y el $63 \%$ de los que dejaron de fumar en la intervención 2, habían hecho uno ó más intentos serios de dejar de fumar en los últimos 5 años.
Puede ser útil incorporar la información sobre el aumento de la probabilidad de éxito a lo largo del seguimiento a materiales escritos de apoyo a la cesación tabáquica. Esta información facilitaría a los médicos mejorar la calidad del consejo para dejar de fumar, al ajustarlo a la situación de los fumadores durante la fase de seguimiento de la intervención.

Por último, es pertinente un comentario sobre el medio donde se ha realizado el estudio. Una gran parte de la población fumadora es accesible en el trabajo por los profesionales sanitarios de la empresa. Los fumadores atendidos en el medio laboral suelen ser más jóvenes, con menor dependencia tabáquica y menor morbilidad que los atendidos en consultas de atención primaria u hospitalaria. Al tratarse de población mayoritariamente sana, pueden prevenirse de forma más eficaz las negativas consecuencias del tabaco sobre la salud. Además, el seguimiento de los sujetos, tal como ha mostrado este estudio, suele ser más completo y fácil que el que se realiza en consultas de atención primaria o en el hospital. Finalmente, además de las razones de salud, hay otras motivaciones importantes para realizar intervenciones médicas de cesación tabáquica en las empresas. La productividad laboral es mayor y el absentismo menor en los exfumadores que en los fumadores $^{25}$.

\section{BIBLIOGRAFÍA}

1. Lancaster T, Stead LF. Individual behavioural counselling for smoking cessation (Cochrane Review). En: The Cochrane Library, Issue 4. Oxford: Update Software; 2001.

2. Silagy C, Stead LF. Physician advice for smoking cessation (Cochrane Review). En: The Cochrane Library, Issue 4. Oxford: Update Software; 2001.

3. Silagy C, Lancaster T, Stead L, Mant D, Fowler G. Nicotine replacement therapy for smoking cessation (Cochrane Review). En: The Cochrane Library, Issue 4. Oxford: Update Software; 2001. 
4. A clinical practice guideline for treating tobacco use and dependence. A US Public Health Service Report. The tobacco use and Dependence Clinical Practice Guideline Panel, Staff, and Consortium Representatives. JAMA 2000;283:3244-54.

5. West R, McNeill A, Raw M. Smoking cessation guidelines for health professionals: an update. Thorax 2000;55:987-99.

6. Grupo de Educación Sanitaria y Promoción de la Salud (ESPS). Guías de educación sanitaria y promoción de la salud del PAPPS. Barcelona: Sociedad Española de Medicina de Familia y Comunitaria, 2000:43-62.

7. Carrión Valero F, Furest Carrasco I, Pascual Izuel JM, Marín Pardo J. Intervención mínima en tabaquismo en España. Arch Bronconeumol 2000;36:124-8.

8. Secker-Walker RH, Chir B, Solomon LJ, Flynn BS, Dana GS. Comparison of the smoking cessation counselling activities of six types of health professionals. Prev Med 1994;23:800-8.

9. Kviz FJ, Clark MA, Prohaska TR, Slezak JA, Crittenden KS, Freels S, Campbell RT. Attitudes and practices for smoking cessation counselling by provider type and patient age. Prev Med 1995; 24:201-12

10. O'Loughlin J, Makni H, Tremblay M, Lacroix Ch, Gervais A, Déry V, Meshefedjian G, Paradis G. Smoking cessation counselling practices of general practitioners in Montreal. Prev Med 2001; 33:627-38.

11. Manley M, Epps RP, Husten C, Glynn T, Shopland D. Clinical interventions in tobacco control: a National Cancer Institute training program for physicians. JAMA 1991;266:3172-3.

12. Norregaard J, Tonnesen P, Petersen L. Predictors and reasons for relapse in smoking cessation with nicotine and placebo patches. Prev Med 1993; 22:261-71.

13. Rodríguez Artalejo F, Lafuente-Urdinguio P, Guallar-Castillón P, Garteizaurrekoa Dublang P, Sáinz Martínez O, Díez Azcárate Ji, Foj Alemán M, Banegas Banegas JR. One-year effectiveness of an individualised smoking cessation intervention at the workplace: a randomised controlled trial. Occup Environ Med 2002. En prensa.

14. Richmond RI, Kehoe LA, Webster IW. Multivariate models for predicting abstention following in- tervention to stop smoking by general practitioners. Addition 1993;88:1127-35

15. Anónimo. Esta es la ayuda eficaz para que consigas dejar de fumar. Barcelona: Novartis Consumer Health; 1997.

16. Heatherton TF, Kozlowski LT, Frecker RC, Fagerström KO. The Fagerström test for nicotine dependence: a revision of the Fagerström Tolerance Questionnaire. Brit J Addict 1991;86:111927.

17. Fleiss JL. Statistical methods for rates and proportions. New York: Johns Wiley \& Sons; 1981.

18. Stapleton JA, Russell MAH, Feyerabend C, Wiseman SM, Gustavsson G, Sawe U, Wiseman D. Dose effects and predictors of outcome in a randomised trial of transdermal nicotine patches in general practice. Addiction 1995;90:31-42

19. Tonnesen P, Paoletti P, Gustavsson G, Russell MA Saracci R, Gulsvik A, Rijcken B, Sawe U. Higher dosage nicotine patches increase one-year smoking cessation rates: results from the European CEASE trial. Eur Respir J 1999;13:238-46.

20. Barrueco M, Torrecilla M, Maderuelo JA, Jiménez Ruiz C, Hernández Mezquita MA, Plaza MD. Valor predictivo de la abstinencia tabáquica a los 2 meses de tratamiento. Med Clin (Barc) 2001; 116:246-50.

21. Regidor E, Gutiérrez-Fisac JL. Indicadores de salud. Cuarta evaluación en España del programa regional europeo Salud para todos. Madrid: Ministerio de Sanidad y Consumo; 1999.

22. Bonfill X, Serra C, López V. Employee and public responses to simulated violations of no-smoking regulations in Spain. Am J Public Health 1997; 87 . 1035-7.

23. Pinto RP, Abrams DB, Monti PM, Jacobus SI. Nicotine dependence and likelihood of quitting smoking. Addictive Behaviors 1987 :12:371-4

24. Richmond RL, Kehoe L, Webster IW. Multivariate models for predicting abstention following intervention to stop smoking by general practitioners. Addiction 1993;88:1127-35.

25. Halpern MT, Shikiar R, Rentz AM, Khan ZM. Impact of smoking status on workplace absenteeism and productivity. Tob Control 2001;10:233-8. 